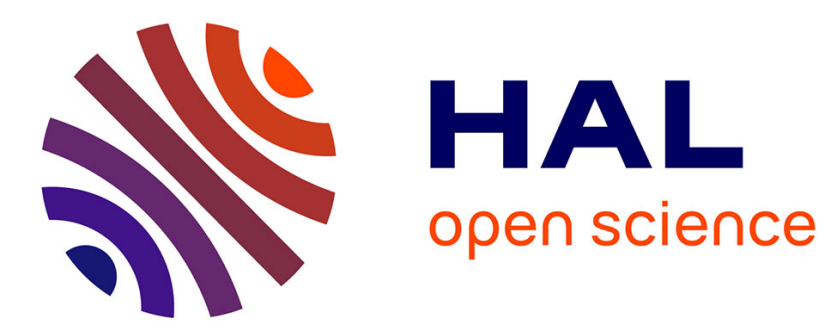

\title{
Multiquarks and Steiner trees
}

J.-M. Richard

\section{- To cite this version:}

J.-M. Richard. Multiquarks and Steiner trees. Nuclear Physics B Proceedings Supplements, 2010, 207, pp.371-374. 10.1016/j.nuclphysbps.2010.10.096 . in2p3-00525240

HAL Id: in2p3-00525240

https://hal.in2p3.fr/in2p3-00525240

Submitted on 11 Oct 2010

HAL is a multi-disciplinary open access archive for the deposit and dissemination of scientific research documents, whether they are published or not. The documents may come from teaching and research institutions in France or abroad, or from public or private research centers.
L'archive ouverte pluridisciplinaire HAL, est destinée au dépôt et à la diffusion de documents scientifiques de niveau recherche, publiés ou non, émanant des établissements d'enseignement et de recherche français ou étrangers, des laboratoires publics ou privés. 


\title{
Multiquarks and Steiner trees*
}

\author{
Jean-Marc Richard ${ }^{\dagger}$ \\ Université de Lyon and Institut de Physique Nucléaire de Lyon \\ Université Claude Bernard-IN2P3-CNRS, 4 rue Enrico Fermi, Villeurbanne, France
}

October 11, 2010

\begin{abstract}
A brief review review is presented of models tentatively leading to stable multiquarks. A new attempt is presented, based on a Steiner-tree model of confinement, which is inspired by by QCD. It leads to more attraction than the empirical colour-additive model used in earlier multiquark calculations, and predict several multiquark states in configurations with different flavours.
\end{abstract}

*Talk given at QCD10, Montpellier, France, June 28 - July 3, 2010

†email: j-m.richard@ipnl.in2p3.fr 


\section{Introduction}

Multiquark spectroscopy is obviously a delicate problem, with regularly experimental candidates that are not confirmed in other experiments [1].

For years, the possibility of multiquarks was mainly discussed in the framework of constituent models already fitting ordinary mesons and baryons. There is a non-trivial technical difficulty when the number of quarks increases from $N=2$ or $N=3$ to $N>3$. But the main problem consists of extrapolating the interaction towards a domain where new colour coupling can be envisaged. In the past, the most current recipe consisted of a pairwise interaction with colour factors. This is justified for the short-range part, but not for the confining part, except when $(N-1)$ of the constituents are closely clustered far from the $N^{\text {th }}$ one. More realistic models have been proposed for many years, and they are now supported by lattice QCD. The linear interaction in quarkonium, understood as a flux tube of minimal length, is generalised as a Steiner-tree linking the constituents through the minimal path.

\section{Binding mechanisms for mul- tiquarks}

Duality The first serious argument in favour of multiquark hadrons was an indirect one, in the context of duality. For a review, see, e.g., [2]. To get consistency in the description of hadronic reactions, a duality principle was imposed relating $s$-channel and $t$-channel exchanges. In baryon-antibaryon scattering, the partners of ordinary mesons are mesons made of two quarks and two antiquarks, preferentially coupled to baryon-antibaryon pairs. In the late 70s and the 80s, such new mesons were tentatively seen in proton-antiproton experiments, but none of baryonium candidates were confirmed in further experiments using better antiproton beams.

Light scalar mesons For light mesons, especially scalars, creating a quark-antiquark pairs does not cost more than providing the existing pair with an orbital excitation [3, \#. This was the beginning of the saga of scalar mesons, which also includes flavour excitation, hybrids, glueballs and various mixing schemes among them. This subject has been discussed in many contributions to this conference.

Chromomagnetism In QCD, the analogue of the Breit-Fermi interaction reads [5] $V_{S S} \propto$ $\sum_{i<j} \delta\left(\boldsymbol{r}_{i j}\right) \tilde{\lambda}_{i} \cdot \tilde{\lambda}_{j} \boldsymbol{\sigma}_{i} . \boldsymbol{\sigma}_{j}$, considered either phenomenological, or given by one-gluon-exchange. There is also a $1 /\left(m_{i} m_{j}\right)$ dependence that reduces the effect for heavy quarks.

At first sight, the colour factor $\tilde{\lambda}_{i} \cdot \tilde{\lambda}_{j}$ simply induces a factor $1 / 2$ for baryons as compared to mesons, and helps to fit the data. However, when combined with the spin factor, it gives a remarkable coherence in some configurations, as first noted by Jaffe [6]. In the $\operatorname{SU}(3)_{\mathrm{f}}$ limit, the expectation value $\left\langle\lambda_{i} . \lambda_{j} \boldsymbol{\sigma}_{i}, \boldsymbol{\sigma}_{j}\right\rangle$ is twice larger for (uuddss) with spin $J=0$ than for the $\Lambda \Lambda$ dissociation threshold.

The di-lambda was first estimated to be deeply bound, since the orbital matrix element $\left\langle\delta\left(\boldsymbol{r}_{i j}\right)\right\rangle$ was assumed to be the same as for ordinary baryons. This assumption, and that of $\mathrm{SU}(3)_{\mathrm{f}}$ symmetry, turn out to give too optimistic estimates. The various corrections work against the stability of the di-lambda 17, 8, 9, 10.

Other configurations receive a coherent attraction of chromomagnetic forces [11], in particular, the 1987-vintage version of the pentaquark [12, 13, 14]. But, again, it turns out very difficult to build a realistic wave function that gives enough short-range correlation for all pairs.

Hadronic molecules It has been often stressed that the conventional strong interactions, which build the deuteron out of two nucleons, can produce other bound states or resonances. There are many contributions to this conference. In particular, a $D \bar{D}^{*}$ composite was predicted, mainly due to onepion-exchange. See the review [15] for references to the original papers. So, when the $X(3872)$ was discovered, it was greeted as a success for this approach. Unfortunately, some of the latest measurements suggest a radial excitation of charmonium, and there is no obvious way to combine the two pictures consistently (see the section on mixing).

In the past, we learned to be careful with the molecular interpretation of the hidden-charm states. When higher $\psi$ resonances were found, theorists were puzzled by the anomalies in the relative decay rates into $D \bar{D}, D^{*} \bar{D}+$ c.c. and $D^{*} \bar{D}^{*}$, and a molecular interpretation was suggested [16] (see, also, [17]). But the branching ratios were later understood from the node structure of the decaying states [18, 19, 20].

Light pentaquark Interesting reviews have been written on the pentaquark episode [21, 22]. The story started with a nice speculation based of chiral dynamics [23], leading Nakano and his colleagues 
to attempt a measurement out of the stream of fashion at that time, and to get a positive signal [24. The real surprise was the wave of positive evidences elsewhere, including experiments having already the data on tape for several years, that nobody had to curiosity to use to search for exotic baryons. However, the pentaquark was not confirmed in high-statistics experiments using sophisticated and expensive detectors with good particle identification.

Some studies were also done in lattice QCD, but somewhat hastily, with a variety of conclusion about the existence of the pentaquark and about its plausible quantum numbers. Clearly, more time would have been required to better distinguish between a genuine bound state or resonance and a state simply occurring from the discretisation of the continuum in the finite volume of the lattice. Better studies are nowadays performed on multiquarks within the framework of QCD sum rules and lattice QCD, as seen in several contributions to this Conference, where references can be found.

Flavour symmetry breaking and chromoelectric binding We adopt here the language of potential models but we believe that the results are much more general. The main advantage of potential models is the possibility of switching on or off some contributions to single out the most effective one for binding.

A remarkable property of the spin-independent interaction among quarks is flavour independence, which induces interesting symmetry breaking effects, and the pattern is similar to that of atomic physics.

For instance, consider the barely bound $\left(e^{+}, e^{+}, e^{-}, e^{-}\right)$molecule, or any rescaled version with the electron mass replaced by another mass $\mu$, and move to configurations involving two different masses. Then it is observed, and proved, that:

- for $\left(M^{+}, m^{+}, M^{-}, m^{-}\right)$: binding deteriorates and is lost for $M / m \gtrsim 2.2$ [25],

- for $\left(M^{+}, M^{+}, m^{-}, m^{-}\right)$: the binding is improved.

But the Coulomb character matters little. What is important, is that the potential does not change when the masses are varied. Hence, a similar behaviour is observed for any four-body problem with flavour independence, with the prediction that in a static and flavour-independent potential, the configuration $(Q Q \bar{q} \bar{q})$ becomes bound if the quark-to-antiquark mass ratio increases.

Explicit quark-model calculations have been carried out to illustrate how this favourable symmetry breaking works with flavour-independent potentials. The corresponding four-body problem is rather delicate, as most other four-body problems. Remember that after Wheeler's proposal in 1945 (the paper was published somewhat later [26]) that the positronium molecule might be stable, a first numerical investigation by Ore [27] concluded that the system is likely unstable, but the following year, Hylleraas and the same Ore published a beautiful analytic proof of the stability [28].

In current quark models, the main conclusion is that binding a doubly-flavoured tetraquark requires a large mass ratio, usually $(b b \bar{q} \bar{q})$ or $(b c \bar{q} \bar{q})$. However, a more sophisticated calculation by Janc and Rosina [29] found $(c c \bar{q} \bar{q})$ barely bound. See, e.g., [30] for a detailed survey of the situation.

\section{Steiner-tree model of confine- ment}

It should be acknowledged, however, that these early constituent-model calculations suffer from a basic ambiguity: how to extrapolate from mesons towards multiquarks. The usual recipe is

$$
V=-\frac{3}{16} \sum_{i<j} \tilde{\lambda}_{i}^{(c)} \cdot \tilde{\lambda}_{j}^{(c)} v\left(r_{i j}\right),
$$

which is presumably justified for the short-range part, but not for the long-range part, except for very peculiar spatial configurations. In (1), the normalization is such that $v(r)$ is the quark-antiquark interaction in ordinary mesons. Strictly speaking, (11) holds for a pairwise interaction with colour-octet exchange. Clearly colour-singlet exchange cannot contribute to confinement, otherwise everything would be confined together, but colour-singlet exchange can contribute to short-range terms. Moreover, there are very likely three-body and multibody forces in baryons and multiquarks.

In the case of baryons, it was suggested very early [31, 32] that the potential generalizing the linear confinement of mesons is

$$
V_{Y}=\sigma \min _{J} \sum_{i=1}^{3} r_{i J}
$$

This was often rediscovered in the context of models (adiabatic bag, flux tubes), or in studies dealing with the strong-coupling regime of QCD [33]. Estimating the baryon energies and properties with the potential (2) is a very interesting 3-body problem. However, the results differ little from these 

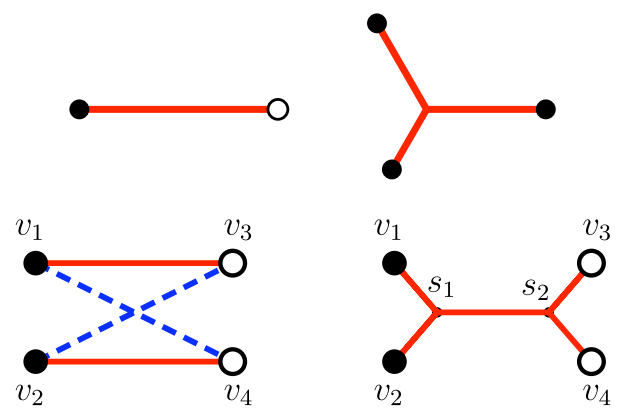

Figure 1: Confinement of mesons and baryons, and tetraquarks. The minimum over the quark permutations gives the flip-flop potential. For the tetraquarks, the minimum is taken of the flip-flop (left) and Steiner tree (right) configurations

obtained using the colour-additive rule, which for baryons reduces to the " $1 / 2$ " rule, namely

$$
V_{3}=\frac{\sigma}{2}\left(r_{12}+r_{23}+r_{31}\right) \text {. }
$$

This $Y$-shape interaction has been generalized to tetraquarks. At first, this looked as an astute guess, but it was later endorsed by detailed lattice QCD [34], including the interplay between flip-flop and connected Steiner tree. See, also, [35] for a study within AdS/QCD. The confining potential reads

$$
\begin{gathered}
U=\min \left\{d_{13}+d_{24}, d_{14}+d_{23}, V_{4}\right\}, \\
V_{4}=\min _{s_{1}, s_{2}}\left(\left\|v_{1} s_{1}\right\|+\left\|v_{2} s_{1}\right\|+\left\|s_{1} s_{2}\right\|\right. \\
\left.+\left\|s_{2} v_{3}\right\|+\left\|s_{2} v_{4}\right\|\right)
\end{gathered}
$$

corresponding schematically to the flux tubes in Fig. 1.

A first study of the tetraquark spectrum with this potential concluded to the "absence of exotics" [36, but a re-analysis by Vijande et al. [37] with a better wave function, indicated that this potential, if alone, and free of constraints due to the Pauli principle, gives stability for the equal-mass case $(q q \bar{q} \bar{q})$, and improved stability for the flavour asymmetric $(Q Q \bar{q} \bar{q})$. It remains to analyse how this stability survives antisymmetrisation, short-range terms in the potential, relativistic effects and spin-dependent corrections. This is however, very encouraging.

The exercise can be repeated for the pentaquark, using the linear model with minimal cumulated length,

$$
\begin{aligned}
V_{P} & =\min \left(V_{\mathrm{ff}}, V_{\mathrm{St}}\right), \\
V_{\mathrm{ff}} & =\min _{i}\left[r_{1 i}+V_{Y}\left(\boldsymbol{r}_{j}, \boldsymbol{r}_{k}, \boldsymbol{r}_{\ell}\right)\right], \\
V_{\mathrm{St}} & =\text { connected Steiner tree },
\end{aligned}
$$
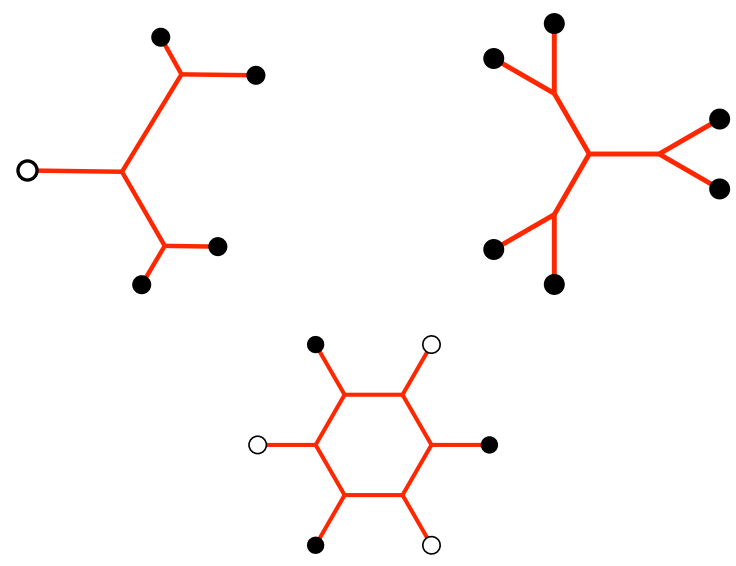

Figure 2: Connected contributions to the confining interaction of a pentaquark, a dibaryon and a baryon-antibaryon system.

the latter, $V_{\mathrm{St}}$, being shown in Fig. 2.

A simple variational calculation gives stability at least for $(\bar{q} q q q q)$, and $(\bar{Q} q q q q)$ and $(\bar{q} q q q Q)$ where $m(Q) \gg m(q)[38]$. The other mass configurations remain to be studied.

This proliferation of stable states in the minimallength model becomes embarrassing. J. Vijande, A. Valcarce and I are now investigating the case of 6-quark configurations. In several cases, the dibaryon $(q q q Q Q Q)$ is found to be stable, as well as the baryonium $(Q Q Q \bar{q} \bar{q} \bar{q})$, but in this latter case, there is a intricate competition between various thresholds: three mesons, a meson and a tetraquark, or a baryon and an antibaryon. When two configurations become degenerate, one expects an enhancement of multiquark binding, provided the variational wave function is flexible enough. Once a stable multiquark is obtained on the basis of this picture of confinement, the role of the neglected effects should be investigated with care, in particular, the shortrange part of the interaction (Coulomb-like forces) and the antisymmetrisation of identical quarks. If the constraint of antisymmetrisation turns out the main obstacle to multiquark stability, then exotic hadrons have to be searched in configurations with quarks of different flavours.

\section{Conclusions}

The problem of multiquark binding is now addressed very seriously with QCD sum rules, Lattice QCD and even AdS-QCD. These ambitious but delicate approaches have first confirmed some results that were previously obtained empirically, such as 
the Steiner-tree structure of the linear term of the quark interaction in the static limit.

The constituent quark model remains a valuable tool of investigation, to detect the most interesting configurations and to analyse the role of the different pieces of the dynamics. In the case of mesons or baryons, the constituent models have been refined over the years, to include relativistic effects, coupling to the continuum, etc. The case of multiquark is of course much more delicate, with the mixing of confined channels and hadron-hadron components probably more crucial to build a reliable wave function.

On the experimental side, it is hoped that the future collider experiments will devote a reasonable amount of time to search for exotics with heavy flavour. As shown by $B$ factories, there is a very good potential of discoveries in hadron physics within experiments primarily designed for studying other aspects of particle physics.

\section{Acknowledgements}

It is a pleasure to thank the organisers of this beautiful Conference for giving us the opportunity of many stimulating discussions.

\section{References}

[1] C. Amsler, et al., Phys. Lett. B667 (2008) 1.

[2] D. P. Roy, Phys. G30 (2004) R113.

[3] R. L. Jaffe, Phys. Rev. D15 (1977) 267.

[4] R. L. Jaffe, Phys. Rev. D15 (1977) 281.

[5] A. De Rujula, H. Georgi, S. L. Glashow, Phys. Rev. D12 (1975) 147-162.

[6] R. L. Jaffe, Phys. Rev. Lett. 38 (1977) 195 198.

[7] J. L. Rosner, $\mathrm{Su}(3)$ breaking and the $\mathrm{h}$ dibaryon, Phys. Rev. D33 (1986) 2043.

[8] G. Karl, P. Zenczykowski, H dibaryon spectroscopy, Phys. Rev. D36 (1987) 2079.

[9] S. Fleck, C. Gignoux, J. M. Richard, B. Silvestre-Brac, Phys. Lett. B220 (1989) 616-622.

[10] T. Sakai, K. Shimizu, K. Yazaki, H dibaryon, Prog. Theor. Phys. Suppl. 137 (2000) 121-145.

[11] B. Silvestre-Brac, J. Leandri, Phys. Rev. D45 (1992) 4221-4239.

[12] H. J. Lipkin, Phys. Lett. B195 (1987) 484.

[13] C. Gignoux, B. Silvestre-Brac, J. M. Richard, Phys. Lett. B193 (1987) 323.

[14] H. J. Lipkin, Nucl. Phys. A625 (1997) 207219.
[15] E. S. Swanson,Phys. Rep. 429 (2006) 243 405.

[16] A. De Rujula, H. Georgi, S. L. Glashow, Phys. Rev. Lett. 38 (1977) 317.

[17] M. B. Voloshin, L. B. Okun, JETP Lett. 23 (1976) 333-336.

[18] A. Le Yaouanc, L. Oliver, O. Pene, J. C. Raynal, Phys. Lett. B71 (1977) 397.

[19] A. Le Yaouanc, L. Oliver, O. Pene, J. C. Raynal, Phys. Lett. B72 (1977) 57.

[20] E. Eichten, K. Gottfried, T. Kinoshita, K. D. Lane, T.-M. Yan, Phys. Rev. D21 (1980) 203.

[21] A. S. B. Tariq, PoS LAT2007 (2007) 136.

[22] C. G. Wohl, in [1]].

[23] D. Diakonov, V. Petrov, M. V. Polyakov, Z. Phys. A359 (1997) 305-314.

[24] T. Nakano, et al., Phys. Rev. Lett. 91 (2003) 012002.

[25] D. Bressanini, M. Mella, G. Morosi, Phys. Rev. A 57 (6) (1998) 4956-4959.

[26] J.A. Wheeler, Ann. N.Y. Acad. Sci. 48 (1946) 219.

[27] A. Ore, Phys. Rev. 70 (1-2) (1946) 90.

[28] E. A. Hylleraas, A. Ore, Phys. Rev. 71 (8) (1947) 493-496.

[29] D. Janc, M. Rosina, Few Body Syst. 35 (2004) 175-196.

[30] J. Vijande, A. Valcarce, N. Barnea, Phys. Rev. D79 (2009) 074010.

[31] X. Artru,Nucl. Phys. B85 (1975) 442.

[32] H. G. Dosch, V. F. Muller, Nucl. Phys. B116 (1976) 470.

[33] T. T. Takahashi, H. Matsufuru, Y. Nemoto, H. Suganuma, Phys. Rev. Lett. 86 (2001) 1821.

[34] F. Okiharu, H. Suganuma, T. T. Takahashi,Phys. Rev. D72 (2005) 014505.

[35] O. Andreev, Phys. Rev. D78 (2008) 065007.

[36] J. Carlson, V. R. Pandharipande, Phys. Rev. D43 (1991) 1652-1658.

[37] J. Vijande, A. Valcarce, J. M. Richard, Phys. Rev. D76 (2007) 114013.

[38] J.-M. Richard, Phys. Rev. C81 (2010) 015205. 